\title{
ELECTROMAGNETIC CHARACTERISTICS OF SIMPLE TARGETS EMBEDDED IN CHIRAL MULTILAYER STRUCTURES
}

\author{
Sidnei J. S. Sant'Anna ${ }^{(1,2)}$ \\ J. C. da S. Lacava ${ }^{(2)}$ \\ David Fernandes ${ }^{(2)}$
${ }^{(1)}$ Divisão de Processamento de Imagem, Instituto Nacional de Pesquisas Espaciais Avenida dos Astronautas, 1758, CEP 12227-010, São José dos Campos -SP, Brasil sidnei@dpi.inpe.br \\ ${ }^{(2)}$ Laboratório de Antenas e Propagação, Instituto Tecnológico de Aeronáutica \\ Praça Mal. Eduardo Gomes, 50, CEP 12228-900, São José dos Campos-SP, Brasil \\ \{lacava,david\}@ita.br
}

\begin{abstract}
The scattering properties of a chiral stratified multilayer structure having an printed planar electric dipole are addressed in this paper. In order to obtain the scattered fields the method of moments in spectral domain is applied. The dipole scattering is characterized by the radar cross section $(R C S)$, the polarimetric response, the $\alpha$-angle from Cloude-Pottier's target decomposition theorem and the directivity function. The results point out that all parameters are sensible to variations on the chiral layer admittance. The chiral layer impinges a rotation on the polarization plane of linearly incident wave for all frequency analyzed.
\end{abstract}

Index Terms - Multilayer structure, printed electric dipole, $R C S$, polarimetric response, scattering.

\section{INTRODUCTION}

The great potential and usefulness of SAR (Synthetic Aperture Radar) data for several remote sensing applications have been proved over the past few decades. In addition the development of techniques for extracting information carried out by SAR data have been increased. Usually, these techniques are based on stochastic or electromagnetic models. The aim of electromagnetic modeling is the description of the interaction process between an electromagnetic wave and natural targets. This description is done by quantifying the total scattering of all elements that can compound a given target. In this approach, it is taking into account the geometry and the electric properties of the each scattering element as well as the main characteristics (frequency, polarization, incident and scattering angles, among others) of the incident electromagnetic wave.

Using the electromagnetic modeling, natural targets might then be described through a set of interconnected scattering elements, having different sizes and random spatial distribution and orientation [1]. A stratified layered medium containing some scattering elements is another way used to represent a natural distributed target. It is important to note that the electromagnetic characteristics of each layer will affect the target scattering when the stratified medium is used. The Faraday rotation, which occurs due to the anisotropic characteristic of the ionosphere, is an example of this kind of influence [2]. The chirality is an electromagnetic property that can have its influence observed by the radar data, as affirmed by Cloude in [3], for some vegetation types. However, Krogager in [4] was one the first researches that considered the target chirality effect, when he had introduced the scattering matrix of a helix in his target decomposition theorem. It is noteworthy to mention that the macroscopic effect caused by chirality is the rotation of the polarization plane of linearly polarized incident wave.

In this work is presented a scattering study of an electric dipole printed in a stratified multilayer structure having one chiral layer. This structure is illuminated by an elliptically polarized plane wave at oblique incidence. The analysis is conducted by means of electromagnetic analytical theory, starting from Maxwell's equations until the establishment of the electromagnetic fields that propagate in this structure, including the electromagnetic fields scattered by the stratified structure. The radar cross section $(R C S)$, the polarimetric response $(P R), \alpha$-angle derived from Cloude-Pottier's decomposition and the directivity function are the parameters used in the target electromagnetic characterization.

The paper is organized as follow. In Section 2, the multilayer structure under analysis is described jointly with a brief explanation of the electromagnetic model employed to solve the scattering problem. The scattering parameters used in the electromagnetic characterization are presented in Section 3. The Section 4 is devoted to analyze the results and the conclusions are drawn in Section 5.

Under FAPESP Grants. 


\section{TARGET STRUCTURE}

The structure under investigation, depicted in Fig. 1, is composed of three linear, homogenous layers stacked up in the z-direction. It contains one confined chiral (bi-isotropic and reciprocal medium) layer located between free space (the upper layer) and ground (the lower layer). The lower layer of complex permittivity $\varepsilon_{g}$ and complex permeability $\mu_{g}$ occupies the negative-z region. The chiral layer is characterized by thickness $\ell$, complex permittivity $\varepsilon$, complex permeability $\mu$ and admittance chiral $\zeta$. A rectangular perfect electric conductor is printed on the planar interface between the free space and the chiral layer (interface $z=d$. The conductor sizes $\ell \times w$ (with $\ell$ along $x$ axis), has infinitesimal thickness and will act as a scatter element. The layers are assumed to be infinite along the transversal $x$ and $y$ directions. The development is based on a global right-handed rectangular coordinate system located on the top of the ground layer (interface $z=0$ ) and lying on the $x y$ plane.

The structure is excited through a uniform plane wave elliptically polarized, having oblique incidence defined by $\theta_{i}$ and $\phi_{i}$ angles of a standard spherical coordinate system. The scattering problem is solved using the spectral domain fullwave technique based on method of moments $(\mathrm{MoM})[5,6]$.

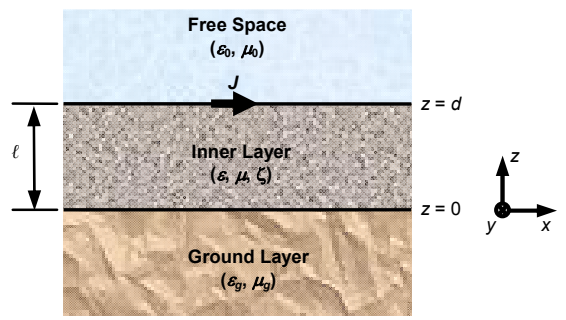

Fig. 1 - Three-layer structure (a lateral view).

In this methodology the structure is treated as a boundary value problem, where the induced current density is the virtual source of the scattered fields. The corresponding spectral Green's functions are obtained analytically in a closed form. Also, knowing the components of the spectral far scattered electromagnetic fields it is possible to obtain the directivity function. The employed approach permits the establishment of asymptotic expressions for the far scattered field components. From these components it is possible to compute several scattering parameters of the structure, such as the radar cross section as well as its scattering matrix [6] and the parameters derived from this matrix. The Sommerfeld-type integrals that appear in MoM are computed using a deformed parabolic path, in order to avoid the integration near the poles of the structure.

\section{SCATTERING PARAMETERS}

The scattering matrix $[S]$ is the most important parameter for electromagnetic target characterization. Basically, it relates linearly the field scattered $\left(\boldsymbol{E}^{s}\right)$ by the target with the field incident $\left(\boldsymbol{E}^{i}\right)$ on it. As consequence the scattering matrix can be seen as a linear operator between $\boldsymbol{E}^{s}$ and $\boldsymbol{E}^{i}$, given by

$$
\left[\boldsymbol{E}^{s}\right]=\left[\begin{array}{c}
E_{v}^{s} \\
E_{h}^{s}
\end{array}\right]=\frac{e^{-i k_{0} r}}{r}\left[\begin{array}{ll}
S_{v v} & S_{v h} \\
S_{h v} & S_{h h}
\end{array}\right]\left[\begin{array}{c}
E_{v}^{i} \\
E_{h}^{i}
\end{array}\right]=\frac{e^{-i k_{0} r}}{r}[S]\left[\boldsymbol{E}^{i}\right],
$$

where vectors are typed in bold face letters.

The $R C S$ is other parameter used for the characterization of target scattering properties. In general, it is function of the operating frequency, wave polarization, incident and observation angles, target geometry and its dielectric properties. The target $R C S$ can be expressed by

$$
R C S\left(\boldsymbol{k}_{s}, \boldsymbol{k}_{i}\right)=\lim _{r \rightarrow \infty}\left[4 \pi r^{2} \frac{\left|\boldsymbol{E}^{s}\left(\boldsymbol{k}_{s}\right)\right|^{2}}{\left|\boldsymbol{E}^{i}\left(\boldsymbol{k}_{i}\right)\right|^{2}}\right],
$$

where $\boldsymbol{E}^{i}\left(\boldsymbol{k}_{i}\right)$ is the electric field of incidence plane wave in $\boldsymbol{k}_{i}$ direction and $\boldsymbol{E}^{s}\left(\boldsymbol{k}_{s}\right)$ is the electric field of the scattered wave in $\boldsymbol{k}_{s}$ direction, measured in a distance $r$ of the target. According to [7] the polarimetric response is another way to represent the target $R C S$. In this graphical representation, the $R C S$ is plotted in function of ellipticity and orientation angles of the scattered electromagnetic wave.

Several parameters have been proposed to assist the interpretation and the classification of polarimetric SAR data. For instance, the entropy, the anisotropy and $\alpha$-angle derived from Cloude-Pottier's target decomposition theorem [8] has been widely used for this purpose. The $\alpha$-angle provides information about the scattering mechanism and for the case in analysis (an electric dipole) it can be estimated from the dominant scatter as

$$
\alpha=\arccos \left\{\frac{\left|S_{v v}+S_{h h}\right|}{\left|\boldsymbol{s}_{3 P}\right|}\right\},
$$

where $\boldsymbol{s}_{3 P}$ is the $[S]$ matrix transformed into the Pauli basis for backscatter case, written as

$$
\boldsymbol{s}_{3 P}=\frac{1}{\sqrt{2}}\left[\left|S_{v v}+S_{h h}\right| e^{i \varphi}\left|S_{v v}-S_{h h}\right| e^{i \delta} \quad 2\left|S_{h v}\right| e^{i \gamma}\right]^{T},
$$

where $\varphi, \delta$ and $\gamma$ are phase angles. Similarly, as described in [9], a general unitary scattering vector can be defined as

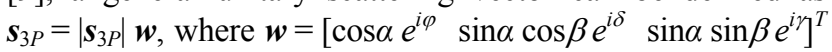
and $\beta$ is the target orientation.

In antenna theory the directivity function $\mathbb{D}(\theta, \phi)$ is one of several parameters used to characterize or define an antenna. This function is defined as the ratio of the radiation intensity $\mathbb{U}(\theta, \phi)$ in a given direction from the antenna to the radiated intensity average over all direction [10].

\section{RESULTS AND DISCUSSION}

The frequencies of 1.25, 5.3 and 9.6 GHz (L-, C- and X-bands) were used in the evaluation of scattering characteristics of the electric dipole. The chiral layer is characterized by a thickness of $32 \mathrm{~mm}, \varepsilon_{r}=2.0, \tan \delta=0.012$ and chiral admittance $(\zeta)$, that for this analysis varied from $0.0 \mathrm{mS}$ to $10.0 \mathrm{mS}$ in step of $0.5 \mathrm{mS}$. The ground layer has $\varepsilon_{r g}=1.0$ and $\tan \delta_{g}=0.12$. The permeability 
$\mu_{0}$ was defined for both layers. An oblique $\left(\theta_{i}=30^{\circ}\right.$ and $\left.\phi_{i}=60^{\circ}\right)$ incident wave having linear polarization (vertical or horizontal) and unitary amplitude is used to excited the dipole. The scattering analysis is conducted based on a particular configuration of the stratified structure, in which it is assumed the existence of scattering elements only in the interface between the free-space region and chiral layer. That is, electric dipole is printed on interface $z=d$ (see Fig. 1) and along the $x$-direction.

Considering the structure of Fig. 1 containing a short dipole, then the function $\mathbb{D}(\theta, \phi)$ is promptly computed. The respective three-dimensional graphic of $\mathbb{D}(\theta, \phi)$ are illustrated in Fig. 2, only in L-band case, for six values of chiral admittance. It can be noted that the pattern as well as the radiation levels are significantly modified by the variation of the inner layer chirality. Besides these cited modifications it is also noted that the chirality impinges a rotation (as already mentioned) on the radiation pattern, as can be seen in Fig. 3. In this figure is shown, for all band, function $\mathbb{D}(\theta, \phi)$ cuts at plane $\theta=45^{\circ}$ for a short electric dipole. From the cuts graphics it is clearly seen the intensity and the rotation variations on the dipole radiation pattern.

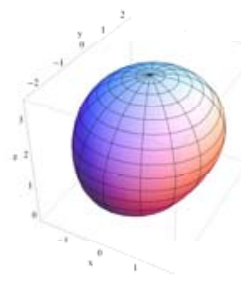

(a) $\zeta=0.0 \mathrm{mS}$

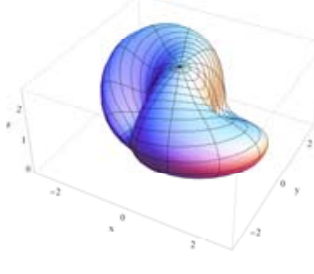

(c) $\zeta=4.0 \mathrm{mS}$

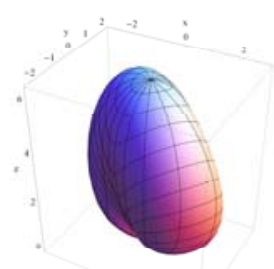

(e) $\zeta=8.0 \mathrm{mS}$

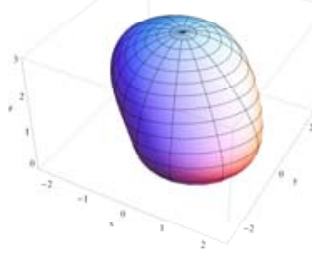

(b) $\zeta=2.0 \mathrm{mS}$

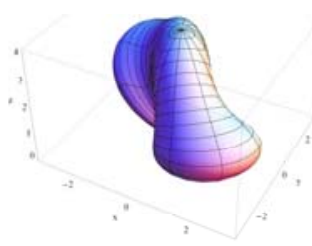

(d) $\zeta=6.0 \mathrm{mS}$

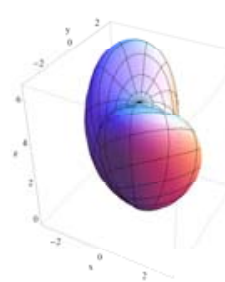

(f) $\zeta=10.0 \mathrm{mS}$
Fig. 2 - Radiation pattern of short electric dipole (L-band).

As aforementioned the far scattered electromagnetic fields for an electric dipole are calculated through MoM. The effect of the $\zeta$ variation in the $R C S$ values was analyzed. For all frequen-

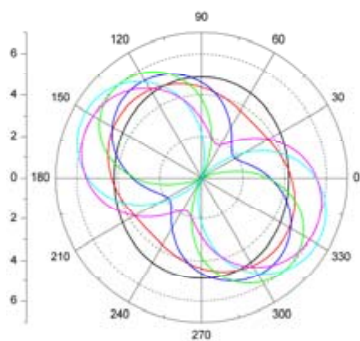

(a) L-band

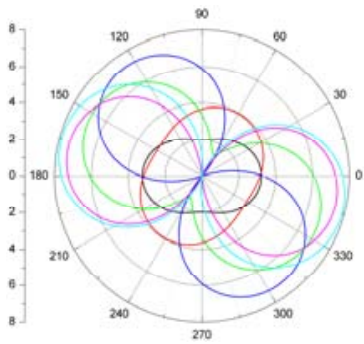

(c) X-band

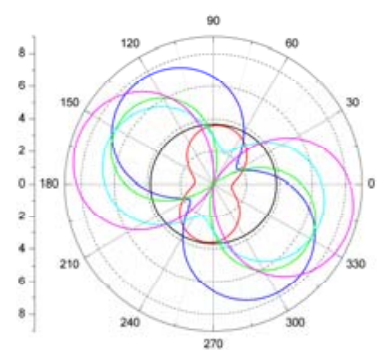

(b) C-band

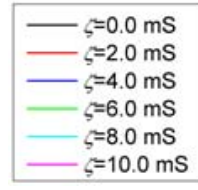

Legend

(d)

Fig. 3 - Function $\mathbb{D}(\theta, \phi)$ cuts at the plane $\theta=45^{\circ}$ for a short electric dipole.

cies it was observed that the $R C S$ values for a horizontally polarized (H-Pol) incident wave are greater than that for V-Pol ones, when the same chiral admittance value is considered. The behavior of $R C S$ is different for each band, presenting different magnitudes and patterns (Fig. 4). In all frequency, when the chirality is included in the model leads to an increasing of the $R C S$ magnitude. In L-band case the chirality did not alter the $R C S$ curves pattern, however they suffer a right shift when the achiral $(\zeta=0.0 \mathrm{mS}) R C S$ curve is used as reference. On the other hand for $\mathrm{C}$ - and $\mathrm{X}$-bands the chirality alters the $R C S$ pattern and this shift is hard to be seen. The mentioned shift is, probably,

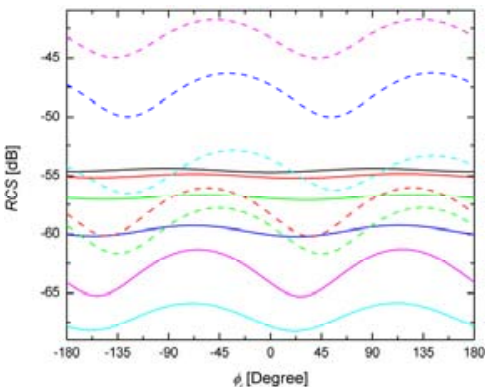

(a) L-band

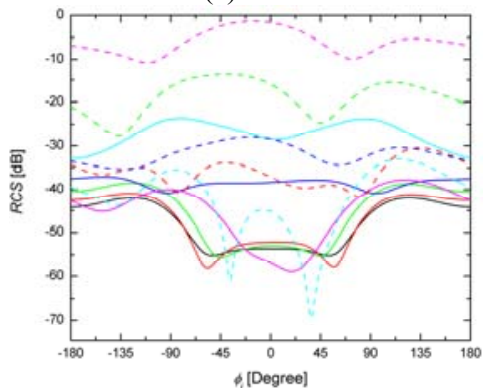

(b) X-band

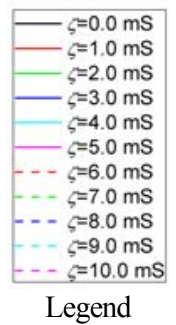

Fig. $4-R C S$ in azimuth for V-Pol incident wave. 
related to the rotation of the polarization plane of linearly incident wave caused by chirality. As an example, the $R C S$ plots for a $\mathrm{V}$-Pol incident wave and in azimuth direction $\left(\theta_{s}\right.$ fixed at $\theta_{i}$ and $-180^{\circ} \leq \phi_{s} \leq+180^{\circ}$ ) are illustrated in Fig. 4, for L- and Xbands.

In Fig. 5 is shown the variation of the $\alpha$-angle in terms of chirality. From $\zeta=3.0 \mathrm{mS}$, in all bands, the $\alpha$-angle is very different from $45^{\circ}$ (black solid line), and it probably occurs due to the existence of cross components in the scattering matrix. It leads to state that the chiralirity introduces a certain degree of anisotropy to the electric dipole scattering mechanism. This anisotropy degree is stronger in C-and X-bands than in L-band.

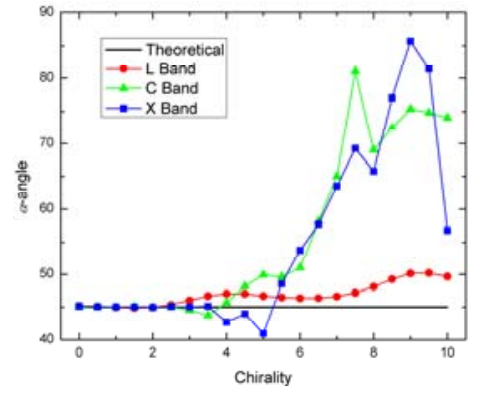

Fig. 5 - The $\alpha$-angle variation in terms of inner layer chirality.

The polarimetric response was also computed and some graphics in co-polarization are depicted in Fig. 6 for two values of chiral admittance, in each band. The polarimetric response, in each

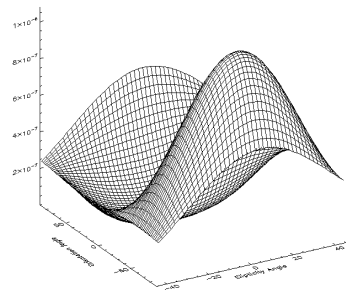

(a) L-band and $\zeta=0.0 \mathrm{mS}$

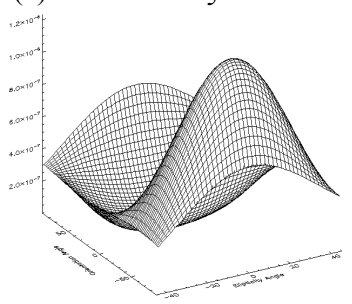

(c) C-band and $\zeta=0.0 \mathrm{mS}$

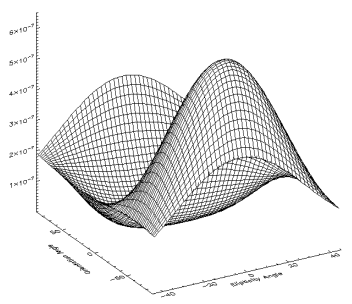

(e) X-band and $\zeta=0.0 \mathrm{mS}$

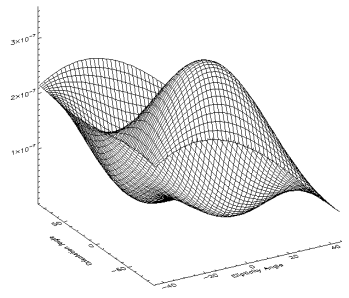

(b) L-band and $\zeta=9.0 \mathrm{mS}$

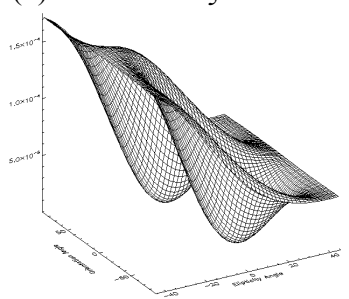

(d) C-band and $\zeta=9.0 \mathrm{mS}$

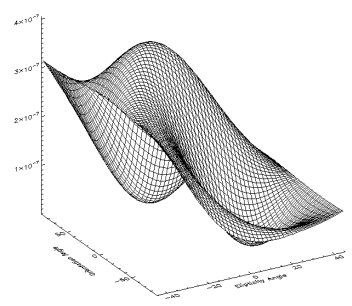

(f) X-band and $\zeta=9.0 \mathrm{mS}$
Fig. 6 - Co-polarized polarimetric response of electric dipole. band, is very sensitive to $\zeta$ variations, since their magnitudes and patterns are altered by changes in chiral admittance. The alteration occurs in a significant way in the polarimetric response pattern, such that the target polarimetric response seems to tend to polarimetric response of a left helix when increasing the chirality.

\section{CONCLUSIONS}

The scattering characterization of an electric dipole embedded in a multilayer structure was carried out by $R C S$, by polarimetric response, by $\alpha$-angle from Cloude-Pottier's target decomposition theorem and by directivity function. It was observed that scattering parameters are sensitive to inner layer chirality variations. This sensitive is highly dependent of the operation frequency. For low values of chiral admittance $(\zeta \leq 3.0 \mathrm{mS})$ and for any band the estimated $\alpha$-angle values are very close to $45^{\circ}$ (typical value for a dipole). That is, from this chirality value the scattering mechanism of the structure can not be considered as being of a dipole. In general, the patterns as well as the levels of the $R C S$ curves are modified by variations in the inner layer chiral admittance. The analysis of polarimetric response showed that increasing the chirality the co-polarization response tends to response of a left helix.

\section{REFERENCES}

[1] C.Y. Lin and K. Sarabandi, "A Monte Carlo coherent scattering model for forest canopies using fractal-generated trees," IEEE Trans. Geosci. Remote Sensing, vol. 37, n. 1, pp. 440-451, 1999.

[2] S.J.S. Sant'Anna, J.C.S. Lacava and D. Fernandes, "Polarization plane rotation effects on SAR polarimetric attributes," Proc. of IGARSS, Cape Town, South Africa, 2009.

[3] S. R.Cloude, "Helicity in radar remote sensing," In Proc. of IGARSS, Toronto, Canada, 2002.

[4] Krogager, E. Aspects of polarimetric radar imaging, $\mathrm{PhD}$ Thesis, Schultz Grafisk A/S, Copenhagen, Denmark, 1993. p. 235.

[5] J.C.S. Lacava, A.V. Proaño De la Torre and L. Cividanes, “A dynamic model for printed apertures in anisotropic stripline structures," IEEE Trans. Microwave Theory and Techniques, vol. 50, n. 1, pp. 22-26, 2002.

[6] S.J.S. Sant'Anna, J.C.S. Lacava and D. Fernandes, "Electromagnetic scattering analysis of simple targets embedded in planar multilayer structures: remote sensing applications," in Advances in Geoscience. and Remote Sens., Gary Jedlovec, Ed., Vukovar: In-Tech, 2009, pp. 619-644.

[7] J. J. van Zyl, H. A. Zebker, and C. Elachi, "Imaging radar polarization signatures: theory and observation". Radio Science, v. 22, n. 4, pp. 529-543, 1987.

[8] S. R. Cloude and E. Pottier, "A review of target decomposition theorems in radar polarimetry". IEEE Trans. Geosc. and Remote Sensing, v. 34, n. 1, pp. 498-512, 1996.

[9] T. Börner, "Methodology for obtaining physical parameters from full polarimetric coherent weather radar data: a first approach to unsupervised Entropy-Alpha-classification". Report for Deliverable 8.3, DLR, 2003.

[10] Balanis, C. A. Antenna theory: analysis and design. New York: John Willey, 1997. p. 941. 